Revista de Matemática: TeORÍA y AplicaCiones 2017 24(1) : 79-96

CIMPA - UCR ISSN: 1409-2433 (PRINT), 2215-3373 (ONLINE)

\title{
OPTIMAL CONTROL PROBLEM FOR A SEIR TYPE MODEL OF EBOLA EPIDEMICS
}

\author{
PROBLEMA DE CONTROL ÓPTIMO PARA UN \\ MODELO DEL TIPO SEIR DE LA \\ EPIDEMIA DEL ÉBOLA
}

\author{
Ellina V. GRigorieva* PAUl B. DeIGNAN ${ }^{\dagger}$ \\ EVGENII N. KHAILOV $\ddagger$
}

Received: 17/Feb/2016; Revised: 5/Aug/2016;

Accepted: 25/Aug/2016

\begin{abstract}
Revista de Matemática: Teoría y Aplicaciones is licensed under a Creative Commons Reconocimiento-NoComercial-Compartirigual 4.0 International License.

Creado a partir de la obra en http://www.revistas.ucr.ac.cr/index.php/matematica

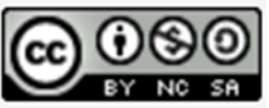

* Department of Mathematics and Computer Sciences, Texas Woman's University, Denton, TX 76204, USA. E-Mail: EGrigorieva@mail.twu.edu

${ }^{\dagger}$ Department of Electrical Engineering, Erik Jonsson School of Engineering and Computer Sciences, The University of Texas at Dallas, Dallas, TX 75080, USA. E-Mail: Paul.Deignan@utdallas.edu

${ }^{\ddagger}$ Department of Computational Mathematics and Cybernetics, Moscow State Lomonosov University, Moscow, 119992, Russia. E-Mail: khailov@cs.msu.su
\end{abstract}




\begin{abstract}
A Susceptible, Exposed, Infectious, and Recovered (SEIR) type control model describing the Ebola epidemic in a population of constant size is considered over a fixed time interval. This model is an extension of the well-known SEIR model and is more suitable to the study of the control mechanism of Ebola epidemics. Along with the traditional SEIR compartments, this model contains an isolated infectious compartment representing the number of infected and exposed individuals that have been isolated from the susceptible individuals. The model has two intervention controls reflecting efforts to protect susceptible individuals from infected and exposed individuals. Additionally, there are two control functions that define efforts for the detection and isolation of infected and exposed individuals. The minimization problem of the sum of total fractions of infected and exposed individuals and total weighted costs of control constraints over a given time interval is stated. For the analysis of the corresponding optimal controls, the Pontryagin maximum principle is used. Accordingly, the controls are bang-bang functions determined by the corresponding switching functions. In order to estimate the number of zeros of the switching functions, a new approach is proposed based on the analysis of the Cauchy problems for the derivatives of these functions. It is found that the optimal controls of the original problem have at most one switching. This allows the reduction of the original complex optimal control problem to the solution of a much simpler problem of conditional minimization of a function of three variables. Results of the numerical solution to this problem and their analysis are provided.
\end{abstract}

Keywords: SEIR model; nonlinear control system; optimal control; Pontryagin maximum principle; switching function.

\title{
Resumen
}

Se considera un modelo de tipo Susceptible, Expuesto, Infeccioso y Recuperado (SEIR) que describe la epidemia del ébola en una población de tamaño constante sobre un intervalo de tiempo fijo. Este modelo es una extensión del bien conocido modelo SEIR y es más adecuado para el estudio del mecanismo de control de la epidemia del ébola. Además de los compartimientos tradicionales del SEIR, este modelo contiene un compartimiento aislado infeccioso que representa el número de individuos infectados y expuestos que han sido aislados de los individuos susceptibles. El modelo tiene dos controles de intervención que reflejan los esfuerzos para proteger a los individuos susceptibles de los individuos infectados y expuestos. Adicionalmente, hay dos funciones de control que definen los esfuerzos para la detección y aislamiento de individuos infectados y expuestos. Se plantea el problema de minimización de la suma del 
total de cocientes de individuos infectados y expuestos y el total de costos ponderados de restricciones de control sobre un intervalo de tiempo. Para el análisis de los correspondientes controles óptimos, se usa el principio del máximo de Pontryanguin. En consecuencia, los controles son funciones bang-bang determinadas por las correspondientes funciones de cambio. Con el fin de estimar el número de ceros de las funciones de cambio, se propone un nuevo enfoque basado en el análisis de los problemas de Cauchy para las derivadas de estas funciones. Se encontró que los controles óptimos del problema original tienen a lo sumo un cambio. Esto permite la reducción del complejo problema original de control óptimo a resolver un problema mucho más simple de minimización condicional de una función de tres variables. Se presentan los resultados y análisis de la solución numérica a este problema.

Palabras clave: modelo SEIR; sistema de control no lineal; control óptimo; principio del máximo de Pontryaguin; función de cambio.

Mathematics Subject Classification: 49J15, 58E25, 92D30.

\section{Introduction}

Ebola is a lethal virus that continues to have significant affect in Central and West Africa. It is transmitted rapidly through direct contact with the infected, their bodily fluids, or tissues. Ebola's high mortality rate and the nonexistence of an effective vaccine make this virus a major problem for public health. Before the time of massive international travel when migration was slow or localized, the virus would not be an intercontinental treat. However, with people all around the world traveling by planes between countries and continents, nobody can be absolutely safe anymore. Indeed, in September 2014 a man infected by Ebola traveling from Africa to Dallas, Texas, and who later died at the Dallas Presbyterian hospital had several contacts in which the disease was spread and temporarily not contained. The infected man died but the nurse who treated him also got infected and was only diagnosed after flying from DFW airport to Washington DC. Ebola was on the USA news for weeks, and the public concern became very high. Government officials were able to stop Ebola from spreading, but only with extraordinary measures.

Since practical interventions rank high in importance of stopping Ebola transmission, it is useful to create mathematical control models describing the dynamics of the Ebola virus spread that includes these measures. It is possible to find the best optimal strategies or preventives measures and corresponding epidemic outcomes by computer simulation of the model with specific initial conditions 
over a given time interval. Motivated by the Dallas Ebola case, we started working on the creation of a mathematical deterministic control model describing the spread of Ebola virus in the affected countries and published two papers [3, 4]. Thus, our paper [3] contains a fairly complete review of works related to mathematical modeling of the spread of Ebola epidemics using SIR and SEIR type compartmental models. This paper is a continuation and further development of our studies presented in [3]. Our goal is to minimize the sum of total fractions of exposed and infected individuals and also the total weighted cost of intervention control constraints over a given time interval.

Let us look closely at the models described in both papers. Both models indeed are of SEIR type describing Ebola epidemics in a population of constant size. However, in this paper, our model has five phase variables and four bounded controls, while paper [3] has four variables and only two controls. The optimal control problem solved in this paper is much more complex. It is notable that the determination of the number of zeros of the switching functions for this model and objective function is a very challenging task.

Once the behavior of the optimal controls is established, the actual numerical solution for determination of the moments of switching is conducted. Our major achievement is that instead of solving a very complex two point boundary value problem for the Pontryagin maximum principle, we have reduced this problem to a much simpler one of constrained minimization and solve it numerically using the overlapping method.

\section{SEIR type model}

Over a given time interval $[0, T]$ we consider a SEIR type model described by the following system of differential equations:

$$
\left\{\begin{array}{l}
\dot{S}(t)=-N^{-1}(\beta I(t)+\alpha E(t)) S(t), \\
\dot{E}(t)=N^{-1}(\beta I(t)+\alpha E(t)) S(t)-(\sigma+\lambda) E(t), \\
\dot{I}(t)=\sigma E(t)-(\gamma+\nu) I(t), \\
\dot{H}(t)=\lambda E(t)+\nu I(t)-\mu H(t), \\
\dot{R}(t)=\gamma I(t)+\mu H(t), \\
S(0)=S_{0}, E(0)=E_{0}, I(0)=I_{0}, H(0)=H_{0}, R(0)=R_{0} ; \\
S_{0}, E_{0}, I_{0}, H_{0}, R_{0}>0 .
\end{array}\right.
$$

Such a model describes the spread of the Ebola epidemic in a population of constant size $N$. Indeed, considering that the equality

$$
S_{0}+E_{0}+I_{0}+H_{0}+R_{0}=N
$$


holds, we add together the equations of system (1). Taking into account relationship (2), we find the equality:

$$
S(t)+E(t)+I(t)+H(t)+R(t)=N .
$$

In system (1) the quantity $S(t)$ is the number of susceptible individuals. The quantity $E(t)$ denotes the number of individuals exposed to the virus who are infected but not yet infectious. Individuals that are infected with the disease and are suffering the symptoms of Ebola are classified as infectious and their number is denoted by $I(t)$. The quantity $H(t)$ denotes the number of individuals that are exposed and infected, but which are isolated from susceptible individuals. Finally, the number of deceased or recovered individuals is denoted by $R(t)$. In system (1) the value $\beta N^{-1} I(t) S(t)$ is the number of individuals infected due to direct contact with an infected individual and the value $\alpha N^{-1} E(t) S(t)$ is the number of individuals infected due to direct contact with an exposed individual. Here $\beta$ and $\alpha$ are the corresponding transmission rates, such that $\beta \geq \alpha$. The value $\sigma E(t)$ is the number of individuals in the exposed stage which show symptoms of the disease and pass on to the infectious stage; $\sigma$ is the infectious rate. The values $\lambda E(t)$ and $\nu I(t)$ are the number of individuals in the exposed and infected stages, which are detected and pass on to the isolated stage; $\lambda$ and $\nu$ are the corresponding detection-isolation rates. The values $\gamma I(t)$ and $\mu H(t)$ are the number of individuals in the infectious and isolated stages which die; $\gamma$ and $\mu$ are the corresponding death rates. We consider that death and recovery are taken to be the same since there has not been a case in which a person who survived Ebola contracts the disease again. Moreover, in the equations of system (1) there are no terms related to natural mortality or fertility because of the short timespan of an Ebola epidemic.

SEIR type models similar to (1), their analysis and use for description of the spread of Ebola epidemics are given for example in [5, 8].

Now, we will create a controllable SEIR type model described by system (1). For this, we introduce two intervention control functions $u(t)$ and $v(t)$ for the efforts of preventing susceptible individuals from becoming infectious individuals as a result of contact with infectious and exposed ones, respectively. Also we introduce two control functions $w(t)$ and $\eta(t)$ that define the efforts on detection and isolation of exposed and infected individuals. For our controls we have the following constraints:

$$
\begin{array}{ll}
0<u_{\min } \leq u(t) \leq u_{\max }, & 0 \leq v_{\min } \leq v(t) \leq v_{\max } \\
w_{\min } \leq w(t) \leq w_{\max }<1, & \eta_{\min } \leq \eta(t) \leq \eta_{\max }<1,
\end{array}
$$

where $u_{\max }=\beta, v_{\max }=\alpha, w_{\min }=\lambda, \eta_{\min }=\nu$. We observe that the control functions $u(t)$ and $v(t)$ regulate the goals and efforts of two similar 
interventions. In the case of control $u(t)$, the equality $u(t)=\beta$ corresponds to not having an intervention affecting the susceptible individuals and the equality $u(t)=u_{\min }$ corresponds to the maximum effort that can be made. Similar behavior at the boundaries is valid for the control $v(t)$. Thus, the equality $v(t)=\alpha$ is where there is no intervention affecting any contacts between the susceptible and exposed individuals and the equality $v(t)=v_{\min }$ is where the maximum effort is made for its prevention. Taking the boundary values for the controls $w(t)$ and $\eta(t)$ has an opposite effect. Thus, if $w(t)=\lambda$ and $\eta(t)=\nu$, then efforts on detection and isolation of exposed and infectious individuals, respectively, are minimal. However, if the corresponding controls take the upper boundaries, $w(t)=w_{\max }, \eta(t)=\eta_{\max }$, then the preventive actions are maximal.

Thus, we have the following SEIR type control model:

$$
\left\{\begin{array}{l}
\dot{S}(t)=-N^{-1}(u(t) I(t)+v(t) E(t)) S(t), t \in[0, T] \\
\dot{E}(t)=N^{-1}(u(t) I(t)+v(t) E(t)) S(t)-(\sigma+w(t)) E(t), \\
\dot{I}(t)=\sigma E(t)-(\gamma+\eta(t)) I(t) \\
S(0)=S_{0}, E(0)=E_{0}, I(0)=I_{0} \\
S_{0}, E_{0}, I_{0}>0 ; S_{0}+E_{0}+I_{0}<N
\end{array}\right.
$$

in which the equation for the function $R(t)$ is excluded, but which can be found from equality (3) by the formula:

$$
R(t)=N-S(t)-E(t)-I(t)-H(t) .
$$

Moreover, we observe from system (1) that the function $H(t)$ is not involved in the first three equations of this system. Also there is no sense to optimize this value. Therefore, in system (5) the differential equation for the function $H(t)$ is excluded as well. This function is obtained as a solution of the following Cauchy problem:

$$
\left\{\begin{array}{l}
\dot{H}(t)=w(t) E(t)+\eta(t) I(t)-\mu H(t), t \in[0, T] \\
H(0)=H_{0}>0
\end{array}\right.
$$

after the controls $w(t)$ and $\eta(t)$ are defined, the functions $E(t)$ and $I(t)$ are found from system (5).

Next, in system (5) we introduce the new variables:

$$
s(t)=N^{-1} S(t), \quad e(t)=N^{-1} E(t), \quad i(t)=N^{-1} I(t)
$$

with corresponding initial values:

$$
s_{0}=N^{-1} S_{0}, \quad e_{0}=N^{-1} E_{0}, \quad i_{0}=N^{-1} I_{0},
$$


for which the following inequalities hold:

$$
s_{0}, e_{0}, i_{0}>0 ; s_{0}+e_{0}+i_{0}<1 .
$$

These variables are the fractions of the quantities $S(t), E(t), I(t)$ in a population of size $N$.

Then, for the variables $s(t), e(t), i(t)$ we obtain the following simpler and more convenient for the subsequent analysis nonlinear control system:

$$
\left\{\begin{array}{l}
\dot{s}(t)=-(u(t) i(t)+v(t) e(t)) s(t), t \in[0, T] \\
\dot{e}(t)=(u(t) i(t)+v(t) e(t)) s(t)-(\sigma+w(t)) e(t) \\
\dot{i}(t)=\sigma e(t)-(\gamma+\eta(t)) i(t) \\
s(0)=s_{0}, e(0)=e_{0}, i(0)=i_{0} \\
s_{0}, e_{0}, i_{0}>0 ; s_{0}+e_{0}+i_{0}<1
\end{array}\right.
$$

For this system the set of all admissible controls $\Omega(T)$ is formed by all possible Lebesgue measurable functions $u(t), v(t), w(t), \eta(t)$, which for almost all $t \in$ $[0, T]$ satisfy constraints (4).

Now, we introduce a region:

$$
\Lambda=\left\{(s, e, i)^{\top} \in \mathbb{R}^{3}: s>0, e>0, i>0, s+e+i<1\right\} .
$$

Here a sign ${ }^{\top}$ means transpose.

The following statement ensures the boundedness, positiveness, and continuation of solutions for system (6).

Proposition 1 If the inclusion $\left(s_{0}, e_{0}, i_{0}\right)^{\top} \in \Lambda$ holds, then for any admissible controls $u(t), v(t), w(t), \eta(t)$ the corresponding solutions $s(t), e(t), i(t)$ for system (6) are defined on the entire interval $[0, T]$ and satisfy the inclusion:

$$
(s(t), e(t), i(t))^{\top} \in \Lambda, \quad t \in(0, T] .
$$

Proof of this fact is standard and so we omit it. Proofs of such statements are given for example in [2,6]. Relationship (7) implies that the region $\Lambda$ is a positive invariant set for system (6).

\section{Optimal control problem}

For control system (6) on the set of all admissible controls $\Omega(T)$ we consider the functional:

$$
J_{1}(u, v, w, \eta)=\int_{0}^{T}(e(t)+i(t)) d t,
$$


which defines the sum of the total fractions of exposed and infected individuals on the given time interval $[0, T]$.

Next, as in [1], we introduce the following costs of intervention and detectionisolation control constraints:

$$
\begin{aligned}
J_{2}(u, v, w, \eta) & =p \int_{0}^{T}((\alpha-v(t)) e(t)+(\beta-u(t)) i(t)) d t \\
& +q \int_{0}^{T}((w(t)-\lambda) e(t)+(\eta(t)-\nu) i(t)) d t,
\end{aligned}
$$

where $p$ and $q$ are the positive weighted coefficients. These costs are linear in the controls $u(t), v(t), w(t), \eta(t)$ and supposed to be proportional to the fractions of the exposed and infected individuals. If one assumes that these fractions are proportional to the fraction of the number of regions where the disease occurs, to the population size, and therefore to the fraction of the number of regions to be converted by our intervention controls to the population size as well. The higher the fractions of exposed and infected, the higher corresponding costs.

Finally, for system (6) on the set of all admissible controls $\Omega(T)$ we consider the optimal control problem of minimization of the sum of values (8) and (9):

$$
\begin{aligned}
& \min _{u(\cdot), v(\cdot), w(\cdot), \eta(\cdot)}\left\{J(u, v, w, \eta)=\int_{0}^{T}[(1+p(\alpha-v(t))+q(w(t)-\lambda)) e(t)\right. \\
&+(1+p(\beta-u(t))+q(\eta(t)-\nu)) i(t)] d t\} .
\end{aligned}
$$

The existence in problem (6), (10) of the optimal controls $u_{*}(t), v_{*}(t), w_{*}(t)$, $\eta_{*}(t)$ and corresponding optimal solutions $s_{*}(t), e_{*}(t), i_{*}(t)$ for system (6) follows from Proposition 1 and Theorem 4 ([7], Chapter 4). This problem and its subsequent analysis are a generalization and further development of the studies presented in [3].

\section{Pontryagin maximum principle}

In order to analyze problem (6), (10), we apply the Pontryagin maximum principle $[9,10]$. According to it, we write the Hamiltonian as

$$
\begin{gathered}
H\left(s, e, i, \psi_{1}, \psi_{2}, \psi_{3}, u, v, w, \eta\right)=(u i+v e) s\left(\psi_{2}-\psi_{1}\right)+\sigma e\left(\psi_{3}-\psi_{2}\right) \\
-w e \psi_{2}-(\gamma+\eta) i \psi_{3}-(1+p(\alpha-v)+q(w-\lambda)) e \\
-(1+p(\beta-u)+q(\eta-\nu)) i
\end{gathered}
$$


where $\psi_{1}, \psi_{2}, \psi_{3}$ are the adjoint variables. Then, for the optimal controls $u_{*}(t)$, $v_{*}(t) w_{*}(t), \eta_{*}(t)$ and corresponding optimal solutions $s_{*}(t), e_{*}(t), i_{*}(t)$ there exists a nontrivial solution $\psi_{*}(t)=\left(\psi_{1}^{*}(t), \psi_{2}^{*}(t), \psi_{3}^{*}(t)\right)^{\top}$ of the adjoint system:

$$
\left\{\begin{aligned}
\dot{\psi}_{1}^{*}(t)= & -\left(u_{*}(t) i_{*}(t)+v_{*}(t) e_{*}(t)\right)\left(\psi_{2}^{*}(t)-\psi_{1}^{*}(t)\right), t \in[0, T], \\
\dot{\psi}_{2}^{*}(t)= & -v_{*}(t) s_{*}(t)\left(\psi_{2}^{*}(t)-\psi_{1}^{*}(t)\right)-\sigma\left(\psi_{3}^{*}(t)-\psi_{2}^{*}(t)\right) \\
& +w_{*}(t) \psi_{2}^{*}(t)+\left(1+p\left(\alpha-v_{*}(t)\right)+q\left(w_{*}(t)-\lambda\right)\right), \\
\dot{\psi}_{3}^{*}(t)= & -u_{*}(t) s_{*}(t)\left(\psi_{2}^{*}(t)-\psi_{1}^{*}(t)\right)+\left(\gamma+\eta_{*}(t)\right) \psi_{3}^{*}(t) \\
& +\left(1+p\left(\beta-u_{*}(t)\right)+q\left(\eta_{*}(t)-\nu\right)\right), \\
\psi_{1}^{*}(T)= & 0, \psi_{2}^{*}(T)=0, \psi_{3}^{*}(T)=0,
\end{aligned}\right.
$$

such that the controls $u_{*}(t), v_{*}(t), w_{*}(t), \eta_{*}(t)$ maximize the Hamiltonian

$$
H\left(s_{*}(t), e_{*}(t), i_{*}(t), \psi_{1}^{*}(t), \psi_{2}^{*}(t), \psi_{3}^{*}(t), u, v, w, \eta\right)
$$

with respect to $u \in\left[u_{\min }, u_{\max }\right], v \in\left[v_{\min }, v_{\max }\right], w \in\left[w_{\min }, w_{\max }\right], \eta \in$ $\left[\eta_{\min }, \eta_{\max }\right]$ for almost all $t \in[0, T]$, and therefore satisfy the relationships:

$$
\begin{aligned}
& u_{*}(t)=\left\{\begin{array}{lll}
u_{\max } & , \text { if } & L_{0}(t)>0, \\
\forall u \in\left[u_{\min }, u_{\max }\right] & , \text { if } & L_{0}(t)=0, \\
u_{\min } & , \text { if } & L_{0}(t)<0,
\end{array}\right. \\
& v_{*}(t)=\left\{\begin{array}{lll}
v_{\max } & , \text { if } & L_{0}(t)>0, \\
\forall v \in\left[v_{\min }, v_{\max }\right] & , \text { if } & L_{0}(t)=0, \\
v_{\min } & , \text { if } & L_{0}(t)<0,
\end{array}\right. \\
& w_{*}(t)=\left\{\begin{array}{lll}
w_{\max } & \text {, if } & L_{w}(t)>0, \\
\forall w \in\left[w_{\min }, w_{\max }\right] & , \text { if } & L_{w}(t)=0, \\
w_{\min } & , \text { if } & L_{w}(t)<0,
\end{array}\right. \\
& \eta_{*}(t)=\left\{\begin{array}{lll}
\eta_{\max } & \text { if } & L_{\eta}(t)>0, \\
\forall \eta \in\left[\eta_{\min }, \eta_{\max }\right] & , \text { if } & L_{\eta}(t)=0, \\
\eta_{\min } & , \text { if } & L_{\eta}(t)<0 .
\end{array}\right.
\end{aligned}
$$

Here the functions

$$
\begin{aligned}
& L_{0}(t)=\left(\psi_{2}^{*}(t)-\psi_{1}^{*}(t)\right)+p s_{*}^{-1}(t), \\
& L_{w}(t)=-\psi_{2}^{*}(t)-q, \quad L_{\eta}(t)=-\psi_{3}^{*}(t)-q
\end{aligned}
$$

are the switching functions, which define the types of the optimal controls $u_{*}(t)$, $v_{*}(t), w_{*}(t), \eta_{*}(t)$ according to relationships (12)-(15). Moreover, the Hamiltonian, which by formulas (16) is rewritten as

$$
\begin{gathered}
H_{*}(t)=H\left(s_{*}(t), e_{*}(t), i_{*}(t), \psi_{1}^{*}(t), \psi_{2}^{*}(t), \psi_{3}^{*}(t), u_{*}(t), v_{*}(t), w_{*}(t), \eta_{*}(t)\right) \\
=e_{*}(t)\left(v_{*}(t) s_{*}(t) L_{0}(t)+\left(\sigma+w_{*}(t)\right) L_{w}(t)-\sigma L_{\eta}(t)-(1+\alpha p-\lambda q)\right) \\
\quad+i_{*}(t)\left(u_{*}(t) s_{*}(t) L_{0}(t)+\left(\gamma+\eta_{*}(t)\right) L_{\eta}(t)-(1+\beta p-(\gamma+\nu) q)\right),(17)
\end{gathered}
$$


is constant on the given interval $[0, T]$.

Now, using formulas (16), the equations and the initial conditions of system (11), we obtain the following system for the switching functions $L_{0}(t)$, $L_{w}(t), L_{\eta}(t)$ :

$$
\left\{\begin{aligned}
\dot{L}_{0}(t)= & \left(u_{*}(t) i_{*}(t)+v_{*}(t) e_{*}(t)-v_{*}(t) s_{*}(t)\right) L_{0}(t) \\
& -\left(\sigma+w_{*}(t)\right) L_{w}(t)+\sigma L_{\eta}(t)+(1+\alpha p-\lambda q), t \in[0, T], \\
\dot{L}_{w}(t)= & v_{*}(t) s_{*}(t) L_{0}(t)+\left(\sigma+w_{*}(t)\right) L_{w}(t) \\
& -\sigma L_{\eta}(t)-(1+\alpha p-\lambda q) \\
\dot{L}_{\eta}(t)= & u_{*}(t) s_{*}(t) L_{0}(t)+\left(\gamma+\eta_{*}(t)\right) L_{\eta}(t) \\
& -(1+\beta p-(\gamma+\nu) q) \\
L_{0}(T)= & p s_{*}^{-1}(T), L_{w}(T)=-q, L_{\eta}(T)=-q .
\end{aligned}\right.
$$

This system will be the subject of our investigation.

\section{Properties of the switching functions}

Analysis of the first two equations of system (18) leads to the statement.

Proposition 2 For all $t \in[0, T]$ the following equality holds:

$$
\frac{d}{d t}\left(s_{*}(t) L_{0}(t)\right)=-s_{*}(t) \dot{L}_{w}(t) .
$$

From the continuity of the functions $L_{0}(t), L_{w}(t), L_{\eta}(t)$ and their initial conditions in system (18), leads to the statement.

Proposition 3 For the switching functions $L_{0}(t), L_{w}(t), L_{\eta}(t)$ there exists such moment of time $t_{0} \in[0, T)$ that simultaneously the following inequalities are valid:

$$
L_{0}(t)>0, \quad L_{w}(t)<0, \quad L_{\eta}(t)<0, \quad t \in\left(t_{0}, T\right] .
$$

Relationships (12)-(15) and inequalities (20) imply that the formulas below for the optimal controls $u_{*}(t), v_{*}(t), w_{*}(t), \eta_{*}(t)$ are true:

$$
u_{*}(t)=u_{\max }, v_{*}(t)=v_{\max }, w_{*}(t)=w_{\min }, \eta_{*}(t)=\eta_{\min }, t \in\left(t_{0}, T\right] .
$$

The second and third equations of system (18) and its initial conditions and also formulas (21) yield the statement.

Proposition 4 For the derivatives of the switching functions $L_{w}(t), L_{\eta}(t)$ the following equalities hold:

$$
\dot{L}_{w}(T)=-1, \quad \dot{L}_{\eta}(T)=-1 .
$$


By formula (17) and the constancy of the Hamiltonian $H_{*}(t)$, the second and third equations of system (18) and its initial conditions, Propositions 3 and 4, we obtain the statement.

Proposition 5 For all $t \in[0, T)$ the relationship

$$
H_{*}(t)=e_{*}(t) \dot{L}_{w}(t)+i_{*}(t) \dot{L}_{\eta}(t)=H_{*}(T)
$$

is valid, where

$$
H_{*}(T)=-\left(e_{*}(T)+i_{*}(T)\right)<0 .
$$

Using Proposition 5, the corresponding equations of system (18) and arguments from the contradiction, we see that the following statement is true.

Proposition 6 The switching functions $L_{0}(t), L_{w}(t), L_{\eta}(t)$ are not equal to zero on any finite interval of $[0, T]$.

Proposition 6 and relationships (12)-(15) show that the optimal controls $u_{*}(t), v_{*}(t), w_{*}(t), \eta_{*}(t)$ are bang-bang functions taking values $\left\{u_{\min } ; u_{\max }\right\}$, $\left\{v_{\min } ; v_{\max }\right\},\left\{w_{\min } ; w_{\max }\right\},\left\{\eta_{\min } ; \eta_{\max }\right\}$, respectively. Moreover, the controls $u_{*}(t), v_{*}(t)$ switch from maximum values to minimum values and vice versa at the same moments of switching.

\section{Estimating the number of zeros of the switching functions}

We consider new approach for estimating the number of zeros of the switching functions $L_{0}(t), L_{w}(t), L_{\eta}(t)$. It is based on the analysis of the Cauchy problems for the derivatives of the switching functions $L_{w}(t), L_{\eta}(t)$.

In order to obtain the differential equations for the functions $\dot{L}_{w}(t), \dot{L}_{\eta}(t)$, we have to be sure that these functions are differentiable almost everywhere on the interval $[0, T]$. By the second and third equations of system (18), for the differentiability of the functions $\dot{L}_{w}(t), \dot{L}_{\eta}(t)$ it suffices if the controls $u_{*}(t), v_{*}(t)$, $w_{*}(t), \eta_{*}(t)$ are piecewise constant functions. That is, they must have a finite number of switchings on the interval $(0, T)$. In turn, this implies that the corresponding switching functions $L_{0}(t), L_{w}(t), L_{\eta}(t)$ have a finite number of zeros on the interval $[0, T]$. Hence, we assume that the following condition holds.

Condition 1 Let the switching functions $L_{0}(t), L_{w}(t), L_{\eta}(t)$ have a finite number of zeros on the interval $[0, T]$. 
Further we will demonstrate that this condition is correct. Condition 1 ensures that the functions $\dot{L}_{w}(t), \dot{L}_{\eta}(t)$ are differentiable almost everywhere on the interval $[0, T]$.

Now, let us consider the switching function $L_{w}(t)$. Using the second equation of system (18), the derivative of the function $\dot{L}_{w}(t)$ is calculated. Then, in the obtained expression we use formulas (19), (23). Adding the first formula from (22) as the corresponding initial condition, finally we have the Cauchy problem for the function $\dot{L}_{w}(t)$ :

$$
\left\{\begin{array}{l}
\ddot{L}_{w}(t)=a(t) \dot{L}_{w}(t)-\sigma H_{*}(T) i_{*}^{-1}(t), t \in[0, T] \\
\dot{L}_{w}(T)=-1
\end{array}\right.
$$

where $a(t)=\sigma+w_{*}(t)+\sigma e_{*}(t) i_{*}^{-1}(t)-v_{*}(t) s_{*}(t)$.

Integrating Cauchy problem (25), we find the formula:

$$
\dot{L}_{w}(t)=-e^{-\int_{t}^{T} a(\xi) d \xi}+\sigma H_{*}(T) \int_{t}^{T} e^{-\int_{t}^{\kappa} a(\xi) d \xi} i_{*}^{-1}(\kappa) d \kappa, t \in[0, T],
$$

which, by (24), implies that

$$
\dot{L}_{w}(t)<0, \quad t \in[0, T]
$$

Hence, the function $L_{w}(t)$ decreases from value $L_{w}(0)$ to value $L_{w}(T)=-q<$ 0 . Therefore, depending on value $L_{w}(0)$ the switching function $L_{w}(t)$ has no more than one zero on the interval $(0, T)$ :

$$
\begin{aligned}
& \text { if } L_{w}(0) \leq 0, \text { then } L_{w}(t)<0, t \in(0, T] \\
& \text { if } L_{w}(0)>0, \text { then } L_{w}(t) \begin{cases}>0, \text { for } 0 \leq t<\tau_{*}, \\
=0, \text { for } t=\tau_{*}, \\
<0, \text { for } \tau_{*}<t \leq T,\end{cases}
\end{aligned}
$$

where $\tau_{*} \in(0, T)$ is a zero of the function $L_{w}(t)$.

Next, let us consider the switching function $L_{\eta}(t)$. Using the third equation of system (18), the derivative of the function $\dot{L}_{\eta}(t)$ is calculated. Then, in the obtained expression we use formula (19). Adding the second formula from (22) as the corresponding initial condition, finally we have the Cauchy problem for the function $\dot{L}_{\eta}(t)$ :

$$
\left\{\begin{array}{l}
\ddot{L}_{\eta}(t)=\left(\gamma+\eta_{*}(t)\right) \dot{L}_{\eta}(t)-u_{*}(t) s_{*}(t) \dot{L}_{w}(t), t \in[0, T] \\
\dot{L}_{\eta}(T)=-1
\end{array}\right.
$$


Integrating Cauchy problem (29), we obtain the formula:

$\dot{L}_{\eta}(t)=-e^{-\int_{t}^{T}\left(\gamma+\eta_{*}(\xi)\right) d \xi}+\int_{t}^{T} e^{-\int_{t}^{\kappa}\left(\gamma+\eta_{*}(\xi)\right) d \xi} u_{*}(\kappa) s_{*}(\kappa) \dot{L}_{w}(\kappa) d \kappa, t \in[0, T]$,

which, by (26), implies that $\dot{L}_{\eta}(t)<0$ for all $t \in[0, T]$. Hence, the function $L_{\eta}(t)$ decreases from value $L_{\eta}(0)$ to value $L_{\eta}(T)=-q<0$. Therefore, depending on value $L_{\eta}(0)$ the switching function $L_{\eta}(t)$ has no more than one zero on the interval $(0, T)$ :

$$
\begin{aligned}
& \text { if } L_{\eta}(0) \leq 0, \text { then } L_{\eta}(t)<0, t \in(0, T] ; \\
& \text { if } L_{\eta}(0)>0, \text { then } L_{\eta}(t) \begin{cases}>0, \text { for } 0 \leq t<\chi_{*}, \\
=0, \text { for } t=\chi_{*}, \\
<0, \text { for } \chi_{*}<t \leq T,\end{cases}
\end{aligned}
$$

where $\chi_{*} \in(0, T)$ is a zero of the function $L_{\eta}(t)$.

Finally, let us consider the switching function $L_{0}(t)$. By relationships (19) and (26), we see that the inequality $\frac{d}{d t}\left(s_{*}(t) L_{0}(t)\right)>0$ is valid for all $t \in[0, T]$. Hence, the function $\widetilde{L}_{0}(t)=s_{*}(t) L_{0}(t)$ increases from value $\widetilde{L}_{0}(0)$ to value $\widetilde{L}_{0}(T)=p>0$. Therefore, depending on value $\widetilde{L}_{0}(0)$ the function $\widetilde{L}_{0}(t)$ has no more than one zero on the interval $(0, T)$ :

$$
\begin{aligned}
& \text { if } \widetilde{L}_{0}(0) \geq 0, \text { then } \widetilde{L}_{0}(t)>0, t \in(0, T] \\
& \text { if } \widetilde{L}_{0}(0)<0, \text { then } \widetilde{L}_{0}(t) \begin{cases}<0, \text { for } 0 \leq t<\theta_{*}, \\
=0, \text { for } t=\theta_{*}, \\
>0, \text { for } \theta_{*}<t \leq T,\end{cases}
\end{aligned}
$$

where $\theta_{*} \in(0, T)$ is a zero of the function $\widetilde{L}_{0}(t)$. By Proposition 1 , we see that the relationships, similar to (32) and (33), hold for the switching function $L_{0}(t)$. Adding here the results obtained above for the functions $L_{w}(t)$ and $L_{\eta}(t)$, we conclude that Condition 1 is correct.

\section{Types of the optimal controls}

Now, we define the possible types of the optimal controls $u_{*}(t), v_{*}(t), w_{*}(t)$, and $\eta_{*}(t)$. Firstly, formulas (12), (13) together with the relationships, similar to (32) and (33), allow us to make conclusion about possible types of the optimal controls $u_{*}(t), v_{*}(t)$. 
Proposition 7 The optimal controls $u_{*}(t), v_{*}(t)$ simultaneously can be either constant functions of the type:

$$
u_{*}(t)=u_{\max }, \quad v_{*}(t)=v_{\max }, \quad t \in[0, T] ;
$$

or piecewise constant functions with one switching of the type:

$$
u_{*}(t), v_{*}(t)= \begin{cases}u_{\min }, v_{\min } & , \text { for } \quad 0 \leq t \leq \theta_{*}, \\ u_{\max }, v_{\max } & , \text { for } \quad \theta_{*}<t \leq T .\end{cases}
$$

Secondly, formula (14) together with relationships (27), (28) enable us to make conclusion about possible types of the optimal control $w_{*}(t)$.

Proposition 8 The optimal control $w_{*}(t)$ can be either a constant function of the type:

$$
w_{*}(t)=w_{\min }, \quad t \in[0, T] ;
$$

or a piecewise constant function with one switching of the type:

$$
w_{*}(t)=\left\{\begin{array}{lll}
w_{\max } & , \text { for } & 0 \leq t \leq \tau_{*}, \\
w_{\min } & , \text { for } & \tau_{*}<t \leq T .
\end{array}\right.
$$

Finally, formula (15) together with relationships (30), (31) allow us to make conclusion about possible types of the optimal control $\eta_{*}(t)$.

Proposition 9 The optimal control $\eta_{*}(t)$ can be either a constant function of the type:

$$
\eta_{*}(t)=\eta_{\min }, \quad t \in[0, T]
$$

or a piecewise constant function with one switching of the type:

$$
\eta_{*}(t)=\left\{\begin{array}{lll}
\eta_{\max } & , \text { for } & 0 \leq t \leq \chi_{*} \\
\eta_{\min } & , \text { for } & \chi_{*}<t \leq T
\end{array}\right.
$$

\section{Solution of the original problem}

Problem (6), (10) can be solved using the following procedure. For arbitrary values $\theta, \tau, \chi \in[0, T]$ we define the controls $u_{\theta}(t), v_{\theta}(t), w_{\tau}(t), \eta_{\chi}(t)$ as

$$
u_{\theta}(t), v_{\theta}(t)= \begin{cases}u_{\min }, v_{\min } & , \text { for } \quad 0 \leq t \leq \theta \\ u_{\max }, v_{\max } & , \text { for } \quad \theta<t \leq T\end{cases}
$$




$$
w_{\tau}(t)=\left\{\begin{array}{ll}
w_{\max } & , \text { for } 0 \leq t \leq \tau, \\
w_{\min } & , \text { for } \tau<t \leq T,
\end{array} \eta_{\chi}(t)= \begin{cases}\eta_{\max } & , \text { for } \quad 0 \leq t \leq \chi \\
\eta_{\min } & , \text { for } \quad \chi<t \leq T\end{cases}\right.
$$

It is easy to see that the controls $u_{\theta}(t), v_{\theta}(t), w_{\tau}(t), \eta_{\chi}(t)$, defined in this way, include all possible types (34)-(39) of the optimal controls $u_{*}(t), v_{*}(t), w_{*}(t)$, $\eta_{*}(t)$, respectively.

Then, system (6) can be integrated on the interval $[0, T]$ with the controls $u_{\theta}(t), v_{\theta}(t), w_{\tau}(t), \eta_{\chi}(t)$. The functions $e_{\theta, \tau, \chi}(t)$ and $i_{\theta, \tau, \chi}(t)$, which correspond to these controls, should be substituted into the functional $J(u, v, w, \eta)$ (see (10)). This yields

$$
F(\theta, \tau, \chi)=J\left(u_{\theta}, v_{\theta}, w_{\tau}, \eta_{\chi}\right), \quad \theta, \tau, \chi \in[0, T],
$$

which is a function of three variables $\theta, \tau, \chi \in[0, T]$, and hence problem (6), (10) is now reduced to a constrained minimization problem

$$
F(\theta, \tau, \chi) \rightarrow \min _{\theta, \tau, \chi \in[0, T]}
$$

Methods for numerical solution of such problem are well-known [11]. Problem (40) is considerably simpler than optimal control problem (6), (10) and can be solved numerically by using the overlapping method, because the Lipschitz constant of the function $F(\theta, \tau, \chi)$ can be easily found (see [3]). Since the Pontryagin maximum principle is only a necessary optimality condition [10], then the function $F(\theta, \tau, \chi)$ can have local minima different from the global minimum that we need to find. To take this into account, we introduce a grid of points on the set, where variables $\theta, \tau, \chi$ are changed. The partitioning of the grid depends on the Lipschitz constant. Comparing the values of the function $F(\theta, \tau, \chi)$ at the nodes of the grid with each other, we find approximately its local minima. Finally, comparing the obtained results we approximately determine the global minimum, which is the solution of problem (40), and hence the solution of the original problem (6), (10).

\section{Numerical results}

We conducted numerical calculations for the values of the parameters $N, T$, $\alpha, \beta, \sigma, \lambda, \gamma, \nu, \mu$ and initial conditions $S_{0}, E_{0}, I_{0}, H_{0}$ for system (1), and control constraints $u_{\min }, u_{\max }, v_{\min }, v_{\max }, w_{\min }, w_{\max }, \eta_{\min }, \eta_{\max }$ from (4), and also the weighted coefficients $p, q$ from formula (9), which are presented below. These values are adopted from $[3,5]$ and based on actual data of the 
Ebola epidemic in Liberia (2014):

$$
\begin{aligned}
& N=6000, \quad S_{0}=5800, \quad E_{0}=80, \quad I_{0}=100, \quad H_{0}=10, \\
& \alpha=0.225, \quad \beta=0.45, \quad \sigma=0.15873, \quad \lambda=0.03, \quad \gamma=0.178253, \\
& \nu=0.05, \quad \mu=0.02, \quad p=1.0, \quad q=1.0, \quad T=300, \\
& u_{\min }=0.225, \quad u_{\max }=0.45, \quad v_{\min }=0.1125, \quad v_{\max }=0.225, \\
& w_{\min }=0.03, \quad w_{\max }=0.045, \quad \eta_{\min }=0.05, \quad \eta_{\max }=0.075 .
\end{aligned}
$$

Figures 1 and 2 show the graphs of the optimal solutions $S_{*}(t), E_{*}(t), I_{*}(t)$, $H_{*}(t)$ and $R_{*}(t)$. The solution $S_{*}(t)$ is shown as a green curve, $E_{*}(t)$ as blue and $I_{*}(t)$ as a red curves on Figure 1. In turn, the solution $H_{*}(t)$ is shown as a green curve and $R_{*}(t)$ as a blue curve on Figure 2.

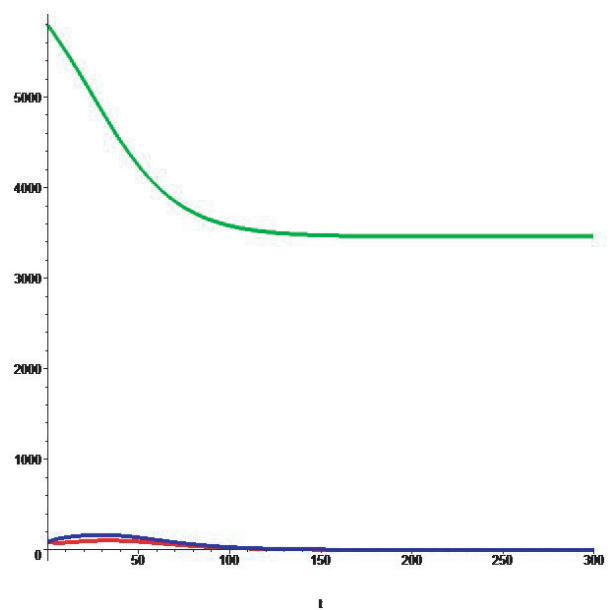

Figure 1: Graphs of the optimal solutions Figure 2: Graphs of the optimal solutions $S_{*}(t), E_{*}(t), I_{*}(t)$.

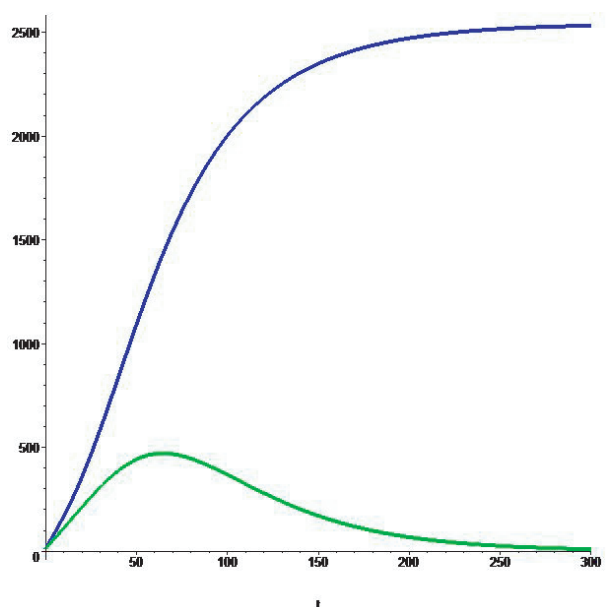

$H_{*}(t), R_{*}(t)$.

The optimal controls $u_{*}(t), v_{*}(t), w_{*}(t), \eta_{*}(t)$ have the following behavior. The controls $u_{*}(t)$ and $v_{*}(t)$, reflecting efforts to protect susceptible individuals from infected and exposed individuals, respectively, take the minimum values $u_{\min }, v_{\min }$ over 297 days during the considered time interval of 300 days. So active intervention control measures should be conducted almost throughout the entire time interval. Switching occurs at the end of this time so that over the last three days the controls $u_{*}(t), v_{*}(t)$ take maximum values $u_{\max }, v_{\max }$, which means the absence of such measures. In turn, the controls $w_{*}(t), \eta_{*}(t)$, defining efforts for the detection and isolation of infected and exposed individuals, respectively, take maximum values $w_{\max }, \eta_{\max }$ during 298 days. This corresponds 
to conducting active detection-isolation measures. Then, again switching occurs, and during the last two days, the controls $w_{*}(t), \eta_{*}(t)$ take minimum values $w_{\min }, \eta_{\min }$, which means the absence of such measures. The behavior of the optimal controls $u_{*}(t), v_{*}(t), w_{*}(t), \eta_{*}(t)$ is explained by the fact that the weighted coefficients $p, q$ from formula (9), determining the relative importance of the functional $J_{2}(u, v, w, \eta)$, are positive. Numerical calculations show that the increase of the values of these coefficients reduces the moments of switching of these controls and vice versa.

\section{Conclusion}

In this paper, we created and investigated a SEIR type control model describing the spread of an Ebola epidemic in a population of a constant size. The model is a nonlinear, deterministic involving five phase variables and four bounded controls. The optimal control problem of minimization the sum of total fractions of exposed and infected individuals and total weighted costs of intervention and detection-isolation control constraints over a given time interval is stated and solved. Using the Pontryagin maximum principle, the analysis of the optimal solutions is conducted analytically. Based on the established properties of the switching functions we propose a new approach for estimating the number of zeros of these functions and state that the optimal controls are bang-bang. The estimates of the number of switchings of the optimal controls for the original problem were found. This allowed the reduction of the original very complex optimal control problem to a considerably simpler one of a constrained minimization for the function of three variables. Numerical calculations and their analysis are presented. Based on our investigation, active intervention preventive control measures should be conducted almost throughout the entire time interval. This is in agreement with health policies, especially for infectious diseases, such as Ebola, for which no vaccination is available.

\section{Acknowledgments}

E.N. Khailov is supported by the Russian Science Foundation, project 14-11-00539. 


\section{References}

[1] Castilho, C. (2006) "Optimal control of an epidemic through educational compaigns", Electronic Journal of Differential Equations 2006(125): $1-11$.

[2] Gaff, H.; Schaefer, E. (2009) "Optimal control applied to vaccination and treatment strategies for various epidemiological models", Mathematical Biosciences and Engineering 6(3): 469-492.

[3] Grigorieva, E.V.; Khailov, E.N. (2015) "Optimal intervention strategies for a SEIR control model of Ebola epidemics”, Mathematics 3(4): 961-983.

[4] Grigorieva, E.; Khailov, E.N. (2015) "Analytic study of optimal control intervention strategies for Ebola epidemic model", in: C. Bonnet, B. PasikDuncan, H. Ozbay \& Q. Zhang (Eds.) Proceedings of the SIAM Conference on Control and its Applications (CT15): 392-399.

[5] Koya, P.R.; Mamo, D.K. (2015) "Ebola epidemic disease: modelling, stability analysis, spread control technique, simulation study and data fitting", Journal of Multidisciplinary Engineering Science and Technology 2(3): 476-484.

[6] Ledzewicz, U.; Schättler, H. (2011) "On optimal singular controls for a general SIR-model with vaccination and treatment", Discrete and Continuous Dynamical Systems supplement: 981-990.

[7] Lee, E.B.; Markus, L. (1967) Optimal Control Theory. John Wiley \& Sons, New York.

[8] Mamo, D.K.; Koya, P.R. (2015) "Mathematical modeling and simulation study of SEIR disease and data fitting of Ebola epidemic spreading in West Africa", Journal of Multidisciplinary Engineering Science and Technology 2(1): 106-114.

[9] Monroy-Pérez, F. (2015) "El Principio del máximo de la teoría de control óptimo: una perpectiva histórica”, Morfismos 19(2): 1-60.

[10] Pontryagin, L.S.; Boltyanskii, V.G.; Gamkrelidze, R.V.; Mishchenko, E.F. (1962) The Mathematical Theory of Optimal Processes. John Wiley \& Sons, New York.

[11] Vasil'ev, F.P. (2002) Methods of Optimization. Faktorial Press, Moscow. 